\title{
Euphémisation du discours journalistique et cadrage dialogique
}

\author{
Montserrat LÓPEZ DÍAZ \\ Universidade de Santiago de Compostela \\ montserrat.lopez.diaz@usc.es \\ ORCID: 0000-0002-8637-1595
}

\section{Resumen}

Desde la perspectiva socio-discursiva del dialogismo bakhtiniano, examinamos algunos eufemismos que circulan en textos informativos de la prensa digital francófona actual. Estos eufemismos forjan referencias difusas a lo que se dice según la doxa, al tiempo que convocan opiniones y puntos de vista compartidos. En la designación de un objeto, las expresiones eufemísticas resultan ser elementos con ecos externos que los hablantes usan a priori por moderación de cara a los demás. Basándonos en un enfoque semántico y pragmático, veremos cómo se elabora el significado y las palabras se minimizan. Así las cosas, el discurso oscila entre decir lo que ya se ha dicho en otra parte y no decir realmente lo que está en juego en la enunciación. Los eufemismos aparecen, además, desde el punto de vista formal, sin ninguna marca gráfica particular que los aísle del resto del enunciado, tanto si pertenecen al periodista como si se derivan de un discurso referido.

Palabras clave: Eufemismo. Dialogismo Texto periodístico. Doxa. Nominación.

\section{Résumé}

Dans l'optique socio-discursive du dialogisme bakhtinien, nous examinons quelques euphémismes qui circulent dans des textes informatifs de la presse numérique francophone actuelle. Ils tissent des renvois diffus au on-dit de la doxa tout en convoquant des opinions et des vues partagées. Dans la désignation d'un objet, les expressions euphémiques s'avèrent des éléments porteurs d'échos extérieurs que les locuteurs utilisent a priori pour des raisons de retenue à l'égard d'autrui. En nous appuyant sur une démarche sémantique et pragmatique nous verrons comment le sens s'élabore et le propos est minimisé. Le discours oscille de ce fait entre dire ce qui a été dit ailleurs et ne pas vraiment dire ce qui est réellement en jeu dans l'énonciation. En outre, les euphémismes apparaissent formellement sans un marquage gra-

\footnotetext{
* Artículo recibido el 17/10/2019, aceptado el 1/04/2020.
} 
phique particulier qui les isole du reste de l'énoncé, tant s'ils appartiennent au journaliste que s'ils dérivent d'un discours rapporté.

Mots-clés : Euphémisme. Dialogisme. Texte de presse. Doxa. Nomination

\begin{abstract}
In the socio-discursive perspective of Bakhtinian dialogism, we examine some euphemisms that circulate in informative texts of the current French-language digital press. They build diffuse references to what is said in the doxa, summoning shared opinions and views. In the designation of an object, the euphemistic expressions turn out to be elements carrying external echoes that the speakers use a priori for reasons of self-control with respect to others. By relying on a semantic and pragmatic approach, we will see how the meaning evolves and the subject is minimized. Thus, the discourse oscillates between saying what has been said elsewhere and not really saying what is really at stake in discourse. In addition, the euphemisms appear formally without any distinctive graphic mark which isolates them from the rest of the utterance, whether they belong to the journalist or they derive from a reported speech.
\end{abstract}

Keywords: Euphemism. Dialogism. Press text. Doxa. Naming.

\title{
1. Introduction
}

L'euphémisme a été défini par la rhétorique comme une "figure de pensée par laquelle on adoucit l'expression d'une idée jugée brutale ou trop amère » (Morier, 1998 : 480). Issu d'une difficulté à nommer quelque chose qui pourrait heurter, il épargne "une désignation attendue tenue pour normale à l'égard du référent " (Détrie, Siblot, Vérine, 2001 : 116) et va de ce fait véhiculer des éléments linguistiques saillants qui pourront bien entendu, le cas échéant, attirer l'attention du destinataire ou, au contraire, rester lettre morte si celui-ci ne les remarque pas.

En tant qu'énonciation adoucie, l'euphémisation mitige la représentation du réel, la rendant moins accessible et actionnant la minimisation de l'éventuel effet négatif de l'acte de parole. Aussi les réalités embarrassantes, avec leurs dénominations propres et habituelles ${ }^{1}$, peuvent-elles voir celles-ci remplacées, le moment venu, par des circonlocutions neutres ou valorisantes qui orientent le discours vers une vision plus positive de la réalité. Cela permet également de déceler certains aspects de l'idéologie et de la mentalité d'une époque, de ses transformations sociales, laissant leur empreinte sur la langue.

Dans la perspective socio-discursive bakhtinienne, le dialogisme est un principe général qui imprègne toute la langue :

Le mot n'est pas une chose, c'est le milieu toujours dynamique, toujours changeant, dans lequel s'effectue l'échange dialogique.

\footnotetext{
${ }^{1}$ Ce sont en l'occurrence les mots tabous ou dysphémiques (Allan \& Burridge, 1991 : 151) mis en quarantaine grâce à l'euphémisme.
} 
Il ne se satisfait jamais d'une seule conscience, d'une seule voix. La vie du mot c'est son passage d'un locuteur à un autre, d'un contexte à un autre, d'une collectivité sociale, d'une génération à une autre. Et le mot n'oublie jamais son trajet, ne peut se débarrasser entièrement de l'emprise des contextes dont il a fait partie.

Tout membre d'une collectivité parlante trouve non pas des mots neutres [....], libres des appréciations et des orientations d'autrui, mais des mots habités par des voix autres. Il les reçoit par la voix d'autrui, emplis de la voix d'autrui. Tout mot de son propre contexte provient d'un autre contexte, déjà marqué par l'interprétation d'autrui. Sa pensée ne rencontre que des mots déjà occupés (Bakhtine 1970 : 263).

Sur cette élaboration incessante des propos des locuteurs sur le fond du déjàdit, Vion (2010 : 2) conclut également, plus récemment, que «le principe dialogique postule que les discours d'un locuteur sont habités de discours antérieurs, de voix qui résonnent dans sa parole et qui constituent le background culturel et idéologique permettant la communication entre les humains ».

L'hypothèse sous-jacente à notre réflexion est que tant le tabou (ou dysphémisme) que l'euphémisme s'avèrent foncièrement "habités ", " occupés ", mais pour des raisons contraires : étant le mot interdit, quelle que soit la cause, et partant chargé et négatif, le tabou pourra être banni du propos, même s'il s'agit du mot propre ou prévisible, et faire place en conséquence à l'euphémisme neutre ou valorisant. Celui-ci à son tour pourra d'ailleurs être senti dans le discours comme une désignation qui remplace à l'occasion le mot attendu. C'est là son caractère figural, et même si l'euphémisme n'est pas complètement nouveau, il s'avère certainement d'un usage plus récent que le tabou qu'il remplace. Les expressions euphémiques s'inscrivent ainsi dans ce principe dialogique général et apparaissent comme des éléments porteurs d'échos extérieurs et antérieurs au discours que les locuteurs utilisent à l'égard d'autrui pour des raisons de retenue et de dédramatisation. L'expression euphémique pourra alors évoquer d'autres usages semblables dans lesquels elle se prête à l'atténuation, tout comme des occurrences où elle ne possède pas de rôle adoucisseur particulier. Cela dit, cette expression ne saurait nullement être a priori dépréciative, autrement elle ne pourrait pas assurer le cas échéant sa capacité à tempérer le propos.

Le discours oscille alors entre dire ce qui a déjà été dit ailleurs dans des contextes semblables où l'on cherche à estomper le propos - car les euphémismes en question sont pour la plupart assez habituels - et ne pas vraiment dire ce qui est réellement en jeu dans l'énonciation. L'euphémisme participe en effet à un flou, ou tout moins à une opacité du discours, dans la mesure où il dit moins que le mot propre attendu en la circonstance et considéré comme dur. Et pour le destinataire qui saisit 
l'euphémisation, il y a bien entendu une autre désignation que celle-ci vient remplacer.

Selon Bakhtine, non seulement le discours entre en dialogue avec d'autres discours qui l'ont précédé et des discours à venir dont il pressent et prévient les réactions, ce sont là les perspectives interdiscursive et interlocutive du dialogisme, mais il faut encore tenir compte des commentaires du locuteur sur sa propre parole, qui constituent la perspective intralocutive avancée par Bres (2005 : 52-53). Or, ce qui nous intéresse surtout dans cette réflexion, c'est le dialogisme interdiscursif « qui ne se montre pas » et qui " relève des mémoires collectives " (Moirand, 2010 : 6), et, éventuellement, le dialogisme interlocutif, du fait que, calculant la portée de son dire, on euphémise surtout "sous la pression d'autrui et à l'intention d'autrui " (Bonhomme, 2012 : 73). C'est la raison pour laquelle, lors de la production du discours, on peut calculer la réaction possible du destinataire afin que le locuteur s'exprime de manière conséquente. Le destinataire à son tour pourra réagir à l'euphémisme, si tant est qu'il l'ait saisi.

Cette étude ${ }^{2}$ se propose d'explorer le dialogisme ${ }^{3}$ dans l'euphémisme à travers l'analyse qualitative de quelques exemples de la presse numérique francophone actuelle en suivant une démarche sémantique et pragmatique. Les exemples sont issus d'un corpus d'une centaine d'articles datés entre 2013 et 2018, dont certains de la base de données Europresse. Du point de vue formel, les euphémismes apparaissent dans des textes informatifs sans marquage graphique particulier apte à les signaler ${ }^{4}$, qu'ils relèvent du libre choix du journaliste ou que celui-ci reformule ou restitue les propos d'autrui. Ils s'inscrivent par là même dans la trajectoire de la polyphonie convergente au sens de Bonhomme (2012 et 2020), étant donné que les différentes voix qui opèrent dans le discours journalistique euphémique s'avèrent consensuelles.

\section{L'épaisseur dialogique des mots}

On a évoqué l'" épaisseur dialogique " (Moirand, 2007: 138) et le " dialogisme de la nomination" (Siblot, 2001:16) qui occupent le mot dans ce qu'il porte de charge

\footnotetext{
${ }^{2}$ Travail réalisé dans le cadre des projets de recherche FFI2013-42249P et FFI2017-85141P (FEDER, Ministerio de Ciencia, Innovación y Universidades, AEI).

${ }^{3}$ Nous suivons la distinction élaborée par Amossy (2005: 69) entre polyphonie et dialogisme : « Le locuteur est à la fois constitué par la parole de l'autre qui le traverse à son insu (il ne peut dire ni se dire en dehors de la doxa de son temps : c'est le dialogisme), et sujet intentionnel mobilisant les voix et points de vue pour agir sur son allocutaire (c'est la polyphonie) ». À l'instar d'Amossy et puis de Bonhomme (2012 et ici même), le dialogisme s'avère un phénomène global et la polyphonie un fait local. Mais nous ne nous occuperons pas des multiples discussions concernant les rapports entre les deux concepts. On peut se reporter à ce sujet, entre autres, aux travaux de Nowakowska (2005), Bres (2005), Rabatel (2006) et Vion (2010), figurant tous dans la bibliographie.

${ }^{4}$ Nous précisons toutefois que dans cet article les euphémismes étudiés ont été mis en italique par nos soins afin de les rendre plus aisément repérables.
} 
sémantique, de représentations préalables et d'attitude énonciative relativement partagées par les interlocuteurs:

L'acte de nommer est constitutivement dialogique, puisque l'objet a été forcément déjà nommé par d'autres, pour d'autres, et que l'on fait appel pour nommer un nouvel événement à ce qu'on a emmagasiné en mémoire à propos d'autres événements, les mots qu'on a rencontrés dans d'autres situations, tout en tenant compte de ceux à qui on s'adresse (Moirand, $2010: 10)$.

Le mot possède des sens qui proviennent des discours où il a été utilisé, ce qui contribue également à sa polysémie. Il exprime immanquablement un point de vue sur la chose quand il la nomme en discours, autant qu'il fait partie d'un paradigme, puisque "dans la nomination, s'ajoutent aux relations à l'objet nommé les relations aux autres dénominations possibles du même objet " (Siblot, 2001:15).

Si l'on envisage alors, par exemple, le mot réforme quand on se rapporte actuellement au monde du travail, il peut renvoyer à " changement " en vue d'améliorer quelque chose pour le bien de tout le monde ou bien a contrario, si l'occasion s'avère moins propice, qui achemine les salariés à l'instabilité, la précarisation, de mauvaises conditions. Les termes contribuent alors à la formation d'un paradigme, mais cela s'entend, avec des valeurs différentes car le regard porté sur l'objet est tantôt rassurant, tantôt défavorable. Dans l'exemple [1] le vocable réforme apparaît lié à des conséquences négatives pour l'emploi :

[1] Les ordonnances réformant le code du travail n'amèneront que "précarité » et "dumping social ", a estimé ce mercredi François Hommeril, le président de la CFE-CGC [...]. « Nous allons nous retrouver face à une énième réforme libérale du marché du travail, la voiture-balai des fantasmes du Medef, qui amènera de la précarité supplémentaire et du dumping social ", déclare M. Hommeril, dans une interview aux quotidiens régionaux du groupe Ebra ( $L e$ Figaro, 9/08/2017).

La question de la réforme va forcément mobiliser des représentations défavorables sollicitées par le contexte proche en vertu du commentaire qui la met en perspective, fait par le syndicaliste François Hommeril. Force est de constater que les enjeux ne sont pas les mêmes selon le choix convoqué par le lexème, car chaque façon de nommer la chose est modelée par le contexte où elle se trouve et par les discours où elle a été employée, c'est-à-dire par les usages que l'on en a faits (Authier-Revuz, 1984 : 100).

Tout en supportant un point de vue, le mot circule en effet empreint de son passé, alors même que le locuteur a tendance à l'interpréter à partir de son présent à lui. Les circonstances actuelles veulent ainsi que le mot réforme, qui a priori renvoie, 
selon le $T L F i$, à « l'amélioration " d'un service, acquière de nouvelles acceptions, voire en récupère de vieillies ${ }^{5}$, en raison des politiques antisociales dont on fait part maintes fois dans la presse actuelle comme "compression ", "réduction ", "instabilité " du personnel et des dépenses dudit service. Ce qui peut provoquer, à l'instar de ce qu'il est dit dans [1], de la "précarité " et du "dumping social ». C'est d'ailleurs dans l'intervalle entre la mise en avant de l'acception positive du mot et celle négative, tout de même présente ou bien en filigrane, que se place la valeur euphémique, qu'émerge la figure. Autrement, si seulement la valeur positive était convoquée, sans un référent posant un problème, il n'y aurait pas d'euphémisation.

Dans la mesure où le dialogisme repose sur le fait que le discours est porté par d'autres discours et orienté vers d'autres discours, les usages préalables que chaque lexème sédimente, y compris les euphémismes, sont inscrits dans les opinions communes, le savoir collectif, les idées ayant cours dans une société à un moment donné :

La doxa constitue une dimension intrinsèque du dialogisme interdiscursif: la relation que tout énoncé entretient avec les énoncés antérieurs marque l'allégeance de la parole à la doxa, c'est-à-dire aux représentations, opinions, croyances communes. [....] La doxa introduit l'altérité au cœur même de ma parole : le discours diffus et anonyme du on est en moi, il me constitue, et je peux tout au plus en prendre conscience et me débattre avec lui sans jamais parvenir à une utopique extériorité (Amossy, 2005 : 66).

Selon cette doxa, en ce qui concerne réforme, circulent deux discours contraires dans la société également partagés, deux représentations dont les locuteurs peuvent ne pas être strictement identifiables: un discours doxique affirme que les réformes des organisations sont souhaitables parce qu'elles apportent des changements avantageux et l'autre discours réfute cette idée, arguant qu'elles déstabilisent les salariés et précarisent leurs conditions de vie. Ces deux idées coexistent à présent, mais correspondent bien entendu à des "positions interprétatives " et des "idéologies" (Bakhtine, $1970:$ 242) divergentes : ce sont globalement celle des employeurs et celle des salariés ${ }^{6}$. Selon les locuteurs et les circonstances de communication l'une ou l'autre prendra le dessus; stockées dans la mémoire, elles n'attendent qu'à être activées.

\footnotetext{
${ }^{5}$ Ce dictionnaire marque comme vieillie l'acception de réforme signifiant « diminution, restriction [...] des dépenses, du service, du train d'une maison ", ainsi que la "réduction à un moindre nombre des employés d'une administration ".

${ }^{6}$ Aussi le même mot ne dit-il pas forcément la même chose pour les uns et pour les autres. Chaque prise de position se fait l'écho d'une situation sociale et arbore une bataille, certes inégale, pour le pouvoir, car déjà l'imposition de la façon de nommer est du ressort du plus fort (López Díaz, 2016).
} 
Suivant l'approche de la sémantique du stéréotype mise au point par Anscombre (2005 : 78-80), on rappellera que les deux discours opposés relèveraient de la signification du mot. Cette sémantique est basée sur des listes ouvertes de phrases de la langue semblables à celles proposées supra, que les locuteurs sont capables d'attacher au vocable réforme afin d'appuyer un raisonnement. Pouvant varier d'un individu à l'autre, ces affirmations sont parfaitement identifiables par les locuteurs du fait qu'elles représentent des opinions générales, même si elles peuvent éventuellement résulter de propriétés fausses ou contradictoires. Mais en tout cas elles servent à exprimer des points de vue d'individus appartenant à une communauté et à assurer le déroulement du discours et sa compréhension.

Comme la valeur des mots doit toutefois être appréhendée dans l'usage qu'on en fait selon les contextes et les référents visés, nous sommes d'avis qu'il est nécessaire, en plus, d'avoir recours à une sémantique proprement discursive et à la pragmatique afin de rendre compte des nuances du texte, d'autant plus que l'euphémisme, disant à mots couverts, tend à brouiller les contours du réel. La sémantique discursive s'occupe du sens compte tenu des données instables fournies par le discours, "à travers les différentes dimensions dans lesquelles le texte se construit, en relation avec le genre, l'interdiscours et les représentations sociales"; ce qui induit que "le sens d'une unité n'est pas un donné que l'on reprend tel quel, mais résulte nécessairement d'un ajustement " (Lecolle, Veniard \& Guérin, 2018 : 45). Cette sémantique est accompagnée par ailleurs d'une approche pragmatique explorant la minimisation du propos.

\section{Euphémismes dans les textes journalistiques et dialogisme}

Certains linguistes ont déjà décrit le fonctionnement dialogique de l'euphémisme. Celui-ci met en place une ouverture du discours qui l'oriente fondamentalement vers d'autres discours déjà entendus : "l'euphémisme apparaît bien comme l'absorption de la voix d'un interdiscours, voix éminemment socialisée, consensuelle, rompue aux échanges propitiatoires qui règlent les échanges pacifiques " (Jaubert, 2008 : 114). Mais il est en même temps un élément remplaçant qui cherche l'acceptation, voire l'anticipation de l'interprétation de l'autre. De surcroît, au dialogisme interdiscursif s'ajoute ainsi le dialogisme interlocutif: "L'acte d'euphémiser renvoie à un usage social, à un dire d'un autre, à un déjà dit, un dit-par-un-autre-quemoi (doxique : dialogisme interdiscursif) et se fonde sur un travail de réappropriation, de co-construction par l'énonciataire, de sa connivence (dialogisme interlocutif) " (Seoane, $2016: 272$ ).

Il va de soi que les deux dimensions interdiscursive et interlocutive soustendent le fonctionnement de l'euphémisme, et qu'il faut les prendre en considération même si le moment venu on met davantage l'accent sur l'une ou sur l'autre. 
Si on utilise des expressions de prime abord valorisantes ou neutres en tant qu'euphémismes dans un contexte sensible, c'est bel et bien parce qu'elles peuvent s'adosser à des énoncés antérieurs où elles véhiculent ces valeurs. Les mots sont porteurs de sens imputables à d'autres usages préalables euphémiques tout autant que non euphémiques, c'est pourquoi l'euphémisme peut également faire venir à l'esprit le tabou qu'il remplace.

Dans l'extériorité et l'antériorité du discours œuvre d'emblée la "mémoire lexicale » en tant qu'ensemble d'hypothèses, sous forme de représentations, recouvrant toutes les facettes de l'expression, qui « font partie des champs sémiotiques formant la culture d'une société au même titre que le savoir encyclopédique " (Lüdi, 1995 : 96).

Dans les exemples [2] et [3] on voit que l'orientation naturelle vers des discours déjà entendus opère ainsi, lorsque l'euphémisme est du domaine technique et plutôt inhabituel pour l'usager ordinaire à l'instar de l'emploi de déprise agricole pour se référer à l'" abandon des terres ", qui serait une dénomination rude, de même que si l'euphémisme est tout à fait banal comme dans l'utilisation de quitter au lieu de « mourir ». Soit les titres :

[2] Un engagement financier contre la déprise agricole (Le Progrès, 26/01/2014).

[3] Simone Veil nous a quittés (20minutes.fr, 30/06/2017).

Ainsi, le nom déprise saisi comme technique, voire soutenu, et plutôt opaque évoque l'action de se déprendre, l'idée de détachement de quelque chose. L'unité polylexicale déprise agricole n'est pas alors nécessairement en mesure de déclencher un automatisme conceptuel et référentiel chez le locuteur ordinaire. À l'opposé, le verbe quitter signifiant "mourir " est largement partagé et complètement transparent pour quiconque. Il relève pourtant d'un usage communicatif plus tempéré que " mourir ». On ne peut pas soustraire le mot à sa polysémie : quitter pourra dès lors être occupé par sa valeur euphémique contextuelle de départ définitif, mais sans exclure complètement l'idée bien plus émoussée de "prendre congé ", " délaisser " tout simplement en accord avec son aspect itératif. Puisque les mots sont porteurs d'une mémoire, l'expression euphémique, quand bien même elle serait lexicalisée, peut voir activé du moins en partie son sens premier ou peut interagir avec lui, ce qui la rend en général plus lisse que le terme ordinaire. Du coup, malgré la banalisation, l'euphémisme peut amener un point de vue moins affirmatif, moins brutal sur un objet du monde soumis en définitive à la "multi-dénomination" (Constantin de Chanay, 2001).

L'euphémisme traverse les discours, on l'a dit, qu'il soit employé sciemment ou non, et prend sens en tant que stratégie qui cherche à arrondir les angles de la réalité en adoucissant le propos. L'usage d'euphémismes répond en effet à cette nécessité d'omettre un type d'expression commune, habituelle, censée être négative, au profit 
d'une autre plus valorisée et plus facilement acceptable. On imprime alors au discours un profil communicatif aseptique, conformément à l'attitude de réserve du locuteur. Par conséquent, l'emploi d'un euphémisme implique autour du mot en question un discours a priori positif, en dépit du fait qu'il puisse se localiser à proximité du tabou qui le dévoile, voire qu'euphémisme et tabou soient convoqués par le même locuteur (López Díaz, 2018). Ainsi, dans [4], il est dit non seulement que la réforme agit essentiellement en faveur des employeurs, mais aussi qu'elle consiste en une avalanche de mesures pro-employeurs, ce qui démasque sans ambages sa valeur euphémique :

[4] C'est la réforme majeure de ce début de quinquennat. Et sans doute la plus importante des (nombreuses) retouches du code du travail réalisées ces dernières années. Les cinq ordonnances "pour le renforcement du dialogue social », en cours d'adoption définitive par le Parlement, viennent bouleverser en profondeur la législation française, pour l'essentiel en faveur des employeurs. Et vont beaucoup plus loin que les propositions du candidat Macron avancées pendant la campagne présidentielle.

Ligne conductrice de la réforme: "lever les freins à l'embauche", selon la formule régulièrement utilisée par ses promoteurs. Soit les mesures qui, dans le droit français, dissuaderaient les entreprises, non pas de procéder à des recrutements, mais à des licenciements... Car dans l'esprit du gouvernement, la peur de recruter est liée aux difficultés à se séparer d'un salarié. Pour y remédier, le texte s'attache donc, dans une première partie, à simplifier le licenciement (avec des mesures relatives au motif économique et à la procédure), mais aussi à l'encadrer financièrement (en plafonnant les indemnités prud'homales dues en cas de séparation abusive). [.....] Face à cette avalanche de mesures pro-employeurs, la hausse de $25 \%$ des indemnités légales de licenciement (pour les dix premières années d'ancienneté), brandie par le gouvernement comme contrepartie en faveur des salariés, aura du mal à convaincre les organisations syndicales de se ranger du côté de l'exécutif (Libération, 18/01/2018).

En même temps qu'il informe des tenants et aboutissants de la réforme, le journaliste montre comment celle-ci traduit encore - comme dans [1] - deux expériences du monde : celle de ses promoteurs et du gouvernement et celle des syndicats et des salariés. L'euphémisme réforme apporte pourtant une coloration méliorative au discours, ne fût-ce qu'au niveau de la simple désignation de l'objet visé : l'expression édulcorée doit évidemment renvoyer plus ou moins subrepticement à un réel pénible et, au moins dans l'énoncé où elle apparaît, celui-ci doit pouvoir rester un peu moins saisissable. L'usage du désignant euphémique a une incidence sur la perception du réel, peu importe qu'elle soit passagère, puisque son style aseptique donne à voir 
comment on se représente le monde et on le communique, et quel lien on cherche à nouer avec le destinataire.

Dans ce qui suit on va explorer deux types d'euphémisation à l'œuvre dans des noms construits : d'une part celle des noms suffixés en -ion que sont les noms d'action polysémiques modération et restructuration et, de l'autre, celle des substantifs portant des préfixes privatifs contre-performance et insuccès, bâtis vraisemblablement dans un souci de désignation bienveillante.

\subsection{Noms d'action}

À des noms comme modération, restructuration on attache aisément des assertions doxiques, largement partagées par la communauté linguistique et corroborées par le dictionnaire, soulignant que la modération est une vertu à laquelle on devrait se conformer ou qu'une restructuration est menée quand quelque chose n'est pas bien organisé, etc. Il s'agira alors d'explorer des collocations comme modération salariale et plan de restructuration de l'entreprise fonctionnant comme des euphémismes. On commencera toutefois par un exemple où modération salariale n'est pas à interpréter comme une atténuation, car on fait référence bien entendu aux salaires colossaux des PDG et membres des conseils d'administration. Dans l'exemple [5] en effet l'expression est justement à prendre dans son sens littéral valorisant :

[5] La dette de SNCF Réseau atteint 45 milliards d'euros. Ça n'a pas empêché Florence Parly d'y gagner 52.000 euros mensuels avant d'être ministre [...].

Selon l'hebdomadaire [Marianne], en 2016 elle avait touché 365.961 euros net de l'entreprise publique, soit 30496 euros net mensuels. En ajoutant ses revenus complémentaires issus de sa présence aux conseils d'administration de sociétés comme Ingenico, Altran, Zodiac Aerospace ou BPI France pour un total de 139.119 euros, plus quelques milliers d'euros de dividendes perçus de ces sociétés, Florence Parly avait touché au total 505.080 euros net en 2016, ajoute l'hebdomadaire, soit 42.090 euros net mensuels et 413.257 euros net de janvier à juin 2017, soit 68.876 euros net mensuels. [...]

Or « Marianne » rappelle que depuis 2012 et un décret adopté lors du premier été du quinquennat Hollande, il existe un plafond limitant les salaires des dirigeants dans le public.[...] Une limite qui ne prend pas en compte les « jetons » de présence dans les conseils d'administration. [...]

En raison d'une faille dans le décret, les hauts cadres comme Florence Parly ne sont donc pas soumis à cet encadrement mais les PDG sont néanmoins encouragés à la modération salariale. Une consigne supposée s'appliquer à la SNCF, d'autant plus qu'elle traîne une dette de 45 milliards d'euros (L'Obs, 9/10/2017). 
On retrouve le sens habituel positif de modération, relevé, entre autres, dans le $T L F i$, qui présente la modération comme une "qualité ", un "comportement d'une personne qui se tient éloignée de tout excès", en particulier "dans le luxe ou dans les plaisirs ». Des synonymes en sont : discrétion, mesure, réserve, retenue, sagesse, etc. La modération s'oppose alors à l'abus, l'excès, ce qui lui octroie une valeur éthique de conduite qu'on devrait observer, de but à atteindre.

Or l'expression peut, bien entendu, être euphémique quand on dit modération salariale pour évoquer la stagnation et l'abaissement des salaires ordinaires. Il n'est plus question alors de corriger un excès quelconque puisque, envers les travailleurs, la modération salariale les met en danger, sous pression et n'est pas une bonne nouvelle, diton dans [6] où plane l'isotopie ${ }^{7}$ de la menace. Celle-ci est d'autant plus probante que la collocation modération salariale fait partie de groupes nominaux où elle est coordonnée à des lexies renfermant une signification nettement négative : la modération salariale et les coupes budgétaires dans les services publics, ainsi que la modération salariale et le démantèlement du service public.

[6] La classe moyenne belge mise en danger par la modération salariale [titre].

La modération salariale et les coupes budgétaires dans les services publics mettent la classe moyenne belge sous pression, a averti mercredi l'Organisation internationale du travail (OIT) [...].

Tant avant que pendant la crise financière, la Belgique a été préservée de l'érosion de la classe moyenne européenne [...]. La préservation de la classe moyenne belge est, d'après Daniel VaughanWhitehead, la conséquence de plusieurs facteurs stabilisateurs tels qu'une tradition de dialogue social, l'indexation automatique des salaires et un secteur public qui a évité de lourdes économies. [...] Mais, avertit l'auteur de l'étude, la tendance à la modération salariale et le démantèlement du service public mettent cette classe moyenne sous pression, y compris en Belgique. Celle-ci «fait beaucoup appel aux services publics. Une plus petite offre d'accueil des enfants signifie par exemple que les deux parents vont moins travailler et donc disposer de moins de revenus ", illustre-t-il.

Cette pression supplémentaire sur la classe moyenne ne constitue pas une bonne nouvelle, prévient l'OIT. C'est elle « qui finance le modèle social européen et assure la croissance économique ", affirme Daniel Vaughan-Whitehead (Le Vif, 24/11/2016).

\footnotetext{
${ }^{7}$ L'isotopie rend compte de l'homogénéité du discours en ce qu'elle implique un ensemble récurrent de traits sémantiques contribuant à la cohésion du texte et permettant son interprétation (cf. Greimas, 1966 ; Rastier, 1985).
} 
L'expression modération salariale annonce moins que ce qu'elle signifie réellement en vertu du référent, mais que l'euphémisme tend à cacher, tout comme dans [7] où elle évoque les conditions de vie pénibles des travailleurs en raison des très faibles augmentations qui les précarisent. Au référent virtuel positif vient se superposer le référent réel négatif de la situation pénible décrite : les salariés sont contre la modération salariale, qui provoque leur mécontentement, leur colère et s'avère la cause de la suppression de postes, de conditions de vie précaires, etc.

[7] Des salariés de Caterpillar Grenoble en grève contre la modération salariale [titre]

Une cinquantaine de salariés de l'entreprise Caterpillar à Grenoble se sont réunis ce 7 février au matin devant le portail de l'usine pour manifester leur mécontentement. À l'origine de leur colère, la remise en cause des acquis sociaux et les faibles augmentations salariales.

"On nous laisse dans la merde ", estime Patrick Martinez, délégué syndical de la CGT sur le site Caterpillar de Grenoble. Présent ce matin aux alentours de 9 heures aux côtés d'une cinquantaine d'ouvriers révoltés, devant l'usine située rue Léon Blum, celui-ci entendait dénoncer des conditions de travail dégradées. Et appuyer les négociations salariales, actuellement en cours avec la direction de l'entreprise.

Le contexte est particulier puisque l'entreprise grenobloise se trouve contrainte d'investir massivement pour prendre en charge la production jusque-là réalisée sur le site belge de Gosselies, suite à l'annonce en septembre de sa fermeture qui s'accompagne de la suppression de 2000 postes.

De nouvelles dépenses pour le site de Grenoble qui se traduisent, de l'avis du syndicat, par une modération salariale affectant le niveau de vie des salariés. "Carterpillar n'hésite pas à puiser dans les acquis sociaux des ouvriers, qui vivent déjà dans des situations souvent précaires", estime ainsi Patrick Martinez. Et celui-ci prend pour exemple un salarié qui dort chaque soir dans sa voiture (Place Gre'net, 7/02/2017).

Dans sa convocation diffuse de discours antérieurs, l'énoncé euphémique fait entendre non seulement sa voix mais aussi celle des énoncés où le vocable modération a été employé comme euphémisme ou non. Et il est sans doute calculé en même temps, pour le moins d'entrée de jeu, à l'égard du destinataire potentiel, car le locuteur peut facilement imaginer les réactions à ses propos qui, aseptisés, devraient en principe avoir plus de chances d'être acceptés.

Quant à l'emploi de restructuration dans le domaine de l'entreprise, il invite au moins a priori à penser qu'il s'agit bel et bien de quelque chose de souhaitable, 
étant donné qu'on restructure ce qui était au préalable mal structuré. Or, dans les textes de presse, les occurrences de plan de restructuration de l'entreprise peuvent apparaître dans des contextes où elles évoquent des faits pénibles comme la suppression d'emplois, ce qui a comme corollaire la menace pour les salariés, qui vivent dans la crainte et l'incertitude :

[8] La direction de l'entreprise ottintoise CP Bourg menace de supprimer 88 emplois! [titre]

Annoncé vendredi aux employés et ouvriers de la société ottintoise, le plan de restructuration sera bien mis en action. On parle d'au moins 80 emplois menacés. Une nouvelle qui était dans l'air mais qui tombe comme un coup de massue pour les 225 personnes travaillant aux sièges de l'entreprise.

"À demain... peut-être ", une phrase qui s'est répétée à plusieurs reprises à la fin de la journée de travail. Derrière ce trait d'ironie, on sentait une réelle crainte chez tous les travailleurs de CP Bourg, cette entreprise spécialisée dans la fabrication de matériel d'impression.

C'est depuis vendredi que toute cette firme vit dans l'incertitude, suite à l'annonce d'un plan de restructuration de l'entreprise qui pourrait mener à la perte de 88 emplois, au bas mot. Ce lundi, une réunion entre la direction et les différents groupes syndicaux s'est tenue afin d'y voir plus clair et défendre les intérêts de tous les travailleurs (Sudinfo.be, 5/10/2017).

Par surcroît, les noms d'action sont polysémiques en ce qu'ils peuvent dénoter un procès et un état simultanément, ce qui déclenche une ambiguïté, comme dans les deux occurrences de modération salariale de l'exemple [7] dans les énoncés : "Des salariés de Caterpillar Grenoble en grève contre la modération salariale » et " De nouvelles dépenses pour le site de Grenoble qui se traduisent, de l'avis du syndicat, par une modération salariale affectant le niveau de vie des salariés». Ils contiennent une ambiguïté en ce qu'on peut interpréter à chaque fois modération salariale comme renvoyant à un fait soit déjà réalisé soit en cours de réalisation. En revanche dans l'exemple [8] le contexte fait seulement émerger l'idée de procès dans les deux occurrences de restructuration de l'entreprise: "Annoncé vendredi aux employés et ouvriers de la société ottintoise, le plan de restructuration sera bien mis en action " et pareillement dans "C'est depuis vendredi que toute cette firme vit dans l'incertitude, suite à l'annonce d'un plan de restructuration de l'entreprise qui pourrait mener à la perte de 88 emplois, au bas mot ».

Dans le même ordre d'idée, on relève en plus, dans ces nominalisations, une indétermination entretenue par l'absence d'agent effectuant l'action, en accord le cas 
échéant avec l'indifférenciation entre procès et résultat ${ }^{8}:$ on peut ainsi se demander qui modère ou a modéré les salaires et qui restructure ou a structuré l'entreprise. Cela étant, si des indices suffisants ne sont pas toujours apportés par le contexte linguistique, ils peuvent l'être néanmoins par le savoir partagé et la connaissance du monde, et nul n'est exempt de faire des supputations sur une interprétation au détriment d'autres possibles.

\subsection{Noms à préfixe privatif}

Dans les substantifs à préfixes, on repère un autre type d'euphémisme qui n'a rien à voir avec la polysémie, puisqu'un mot valorisant est orienté négativement par le préfixe qu'on lui attribue. L'écho dialogique favorable vient dans ce cas uniquement du mot simple qui sert de base et non d'un sens propre préalable du mot construit.

Le préfixe contre- marque l'opposition, l'inverse symétrique de la chose désignée par la base, comme dans contre-performance. Si on part de la base performance, le discours lexicographique renvoie au résultat obtenu dans une épreuve sportive, et notamment à la victoire, à l'exploit ; et par extension au succès remporté par quelqu'un lors d'une exhibition qui demande des qualités exceptionnelles. Le mot contre-performance est au demeurant défini par le TLFi comme "mauvaise performance, résultat anormalement médiocre ", ce qui traduit du coup l'orientation contraire en une sorte d'exception à la règle préalable que constitue le vocable de base.

Ainsi, selon Dal (2003), le préfixe contre- " dit du référent du dérivé qu'il construit qu'il constitue une réplique du référent de la base à laquelle il s'applique, supposant par la même occasion une occurrence liminaire de ce à quoi réfère la base ", ce qui va dans le sens du "présupposé de secondarité " mis en lumière par Amiot et de Mulder (2003). On ferait dès lors mention d'une contre-performance quand une "performance " a eu lieu préalablement ou a été prévue. Dans le domaine sportif, par exemple, contre-performance est un euphémisme pouvant qualifier aussi bien un match nul en [9] qu'une défaite en [10], mais c'est toujours le présupposé que ce résultat serait anormal ou inattendu qui est sollicité. Cette utilisation en discours concourt du reste à amoindrir sa portée :

[9] Mondial 2018: "C'est une contre-performance», reconnaît Deschamps [titre]

[...] Après ce résultat [0-0]. "On peut toujours mieux faire. On aurait pu mieux faire en première période et il nous a manqué de la qualité technique et de la percussion. En seconde période, on a fait tout ce qu'il fallait mais il nous a manqué l'efficacité. C'est ça aussi le foot ». [...] «En termes de résultat et sur le plan comptable, c'est une contre-performance. Mais on vient de livrer une première bataille et il en reste neuf » [...] (L'Express, 6/09/2016).

\footnotetext{
${ }^{8}$ Sur ce sujet on peut consulter Benetti \& Corminboeuf (2004) et Huyghe (2014).
} 
[10] Gilles Depriester : «Notre première contre-performance de la saison " [titre]

En déplacement chez le dernier du championnat, Harnes, Dunkerque avait toutes les armes pour l'emporter. Breakés de deux sets, les hommes de Gilles Depriester ont finalement affiché un visage bien pâle : 3-1. "On n'a pas fait un gros match et en face ils jouent les points à fond. Ils n'espèrent plus rien dans ce championnat, juste se faire plaisir. C'est une contre-performance mais on n'est qu'au deuxième match retour, donc il y a encore des points à prendre ", restait optimiste le coach maritime, Gilles Depriester (La Voix du Nord, 4/02/2013).

En outre, les exemples [9] et [10] laissent apparaitre des constructions similaires en ceci qu'on qualifie un fait ayant eu lieu comme une contre-performance, et ensuite l'argumentation est inversée au moyen du connecteur mais dans le but de minorer ses dommages : mais on vient de livrer une première bataille et il en reste neuf dans l'exemple [9] et mais on n'est qu'au deuxième match retour dans [10]. Cela contribue, au demeurant, à mettre en avant le caractère accessoire du préfixe contre-, qui ne nie pas déjà intrinsèquement le concept de base avec lequel il se combine9.

En dehors du sport, en économie, ce même euphémisme est aussi productif pour désigner un échec qui fait exception en quelque sorte. Mais, tandis que le caractère "second " que contre-imprime au composé était seulement récupérable en [9] et [10], dans l'exemple suivant il est parfaitement explicite. Dans [11] sont en effet concomitants le terme de base et le dérivé ${ }^{10}$, si bien qu'à l'occurrence du mot performance fait écho quatre phrases après le GN la contre-performance, précédé par surcroît du connecteur d'opposition toutefois. L'ensemble contribue alors à créer l'effet d'exception de ladite contre-performance qui confirme la règle de la progression largement majoritaire du groupe JCDecaux:

[11] Le groupe de communication extérieure JCDecaux a plus que doublé son bénéfice net en 2014[...]. Fin janvier, le numéro 1 mondial du secteur avait publié un chiffre d'affaires annuel record à 2,813 milliards d'euros (3 737 milliards de dollars), en hausse de $5,1 \%$, et une croissance organique atteignant $3,8 \%$ [...]. «Cinq régions sur six sont en progression. L'Europe a repris des couleurs avec une croissance organique de $4,1 \%$ après plusieurs années difficiles, ce qui est une belle performance ", a tenu à souligner $M$.

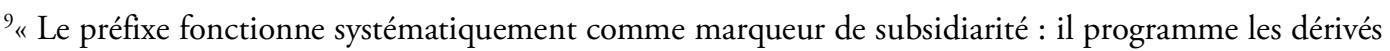
qu'il construit à référer à des entités constituant des répliques à/de ce que désignent les bases sur lesquelles il opère, sans que les répliques en question aient nécessairement pour vocation d'annihiler ou de neutraliser ce à quoi réfèrent les bases " ( $\mathrm{Dal}, 2003)$. On peut consulter également Van Goethem (2006).

${ }^{10} C f$. à ce sujet l'analyse de Dal (2003).
} 
Decaux. La France a connu une croissance «molle » à $0,4 \%$ en organique et le Royaume-Uni a progressé de 1,6\% en cette année post JO. En 2014, l'Asie-Pacifique est devenue la deuxième région géographique du groupe (23,3\% de son chiffre d'affaires), devant la France, et a affiché une croissance organique de $7,3 \%$, portée par la Chine. Les pays à forte croissance représentent désormais un tiers des revenus du groupe. A noter toutefois la contre-performance de l'Amérique du Nord (-5,8 \%) (La Correspondance de la publicité, 6/03/2015).

L'effet de secondarité par rapport à une situation usuelle ou attendue est déjà inhérent au mot contre-performance, sans que le préfixe annule la base, ce qui convient d'ailleurs parfaitement à son fonctionnement euphémique. Lorsque performance est en plus cooccurrent, le caractère anecdotique de son dérivé semble encore davantage sollicité.

Quant au préfixe négatif in-, il sert à indiquer la privation de quelque chose (cf. Staaff, 1928). Insuccès est le manque de succès, de réussite ${ }^{11}$. Le mot est par conséquent un synonyme doux d' "échec ", mot qui a une valeur dénominative plus nette et puissante. Mais tout comme "performance ", "succès " est un mot très positivement connoté, laissant des traces sur insuccès. Celui-ci peut d'ailleurs entrer en concurrence dans certains contextes avec contre-performance et faire ainsi partie du même paradigme, les deux confinés pour l'heure en général aux usages des professionnels. D'après les exemples suivants le mot ne peut émaner que d'un locuteur indulgent :

[12] Tennis: Federer met fin à deux ans d'insuccès en Masters 1000 [titre]

Le Suisse Roger Federer a mis fin à une disette de tout juste deux ans en Masters 1000 en remportant le tournoi de Cincinnati face à l'Espagnol David Ferrer 6-3, 1-6, 6-2, dimanche (L'Express, 18/08/2014).

[13] Des records de fréquentation, une programmation cosmopolite et pointue... Un an après une saison chaotique, marquée par la grève des intermittents, le directeur du festival d'Avignon affiche un bilan radieux, malgré l'insuccès critique de son adaptation trop théorique du Roi Lear (Télérama, 24/07/2015).

[14] Pouvoir d'achat en berne et surtout multiplication des promotions. Telles sont les causes profondes du relatif insuccès des premiers jours des soldes d'hiver. La fréquentation des magasins

${ }^{11}$ Il va sans dire que le sens compositionnel d'insuccès est loin de la polysémie de in-dans certains adjectifs en -able, du fait qu'ils peuvent avoir une valeur compositionnelle ou bien superlative et hyperbolique, celle-ci prenant d'ailleurs le dessus : innommable veut dire ainsi " qu'on ne peut pas nommer ", mais surtout "indigne, dégoûtant ", de même qu' inqualifiable signifie avant tout " odieux " et incroyable « extraordinaire " (cf. Apothéloz, 2003 et 2005; Berlanga de Jesús, 2000). 
constatée lors du premier week-end de 2015 , soit les 10 et 11 janvier, est inférieure de $3,8 \%$ à celles de la même période un an plus tôt dans l'Hexagone (La Tribune, 15/01/2015).

Dans le dialogisme de la nomination, insuccès interagit avec les échos mémoriels de ses emplois préalables pour annoncer moins que "défaite " ou " échec ». Les noms à préfixe privatif contre- et in- signifient la non-existence, le manque de performance et de succès, mais non l'échec, la défaite, voire le désastre, la catastrophe, puisque la base positive reste tout de même dans le nouveau mot et, ne fût-ce que de manière diffuse, dans la mémoire des locuteurs.

Il est à rappeler en effet que « les rapports mémoriels fonctionnent souvent à l'insu des locuteurs et que les mémorisations échappent partiellement à la conscience des sujets » (Moirand, 2007 : 134); cela en vertu de l'action des usages des mots sur leur interprétation. De tels propos minorés inscrivent en eux-mêmes des discours autres, y compris le discours social, la vox populi, qui agissent au niveau de leur charge sémantique et de leur potentiel pragmatique.

\section{Conclusion}

Dans son épaisseur dialogique, et en particulier sa dimension doxique, l'euphémisme renvoie au déjà-dit, en le confrontant à d'autres emplois aussi divers que les possibilités du mot, et au non-dit, car il est aussi opposé au tabou ou dysphémisme qu'il remplace. Comme il ressort des exemples examinés, sans marquage graphique distinctif ni commentaire, l'euphémisme se fait simplement remarquer dans un réseau de connexions interdiscursives par rapport à l'objet qu'il désigne et par rapport à d'autres désignations possibles que celui-ci pourrait accueillir, mais également aux emplois préalables de l'expression en question. Ce sont des façons de dire parfois relativement peu répandues car circonscrites à des milieux professionnels, parfois assez communes puisque popularisées auprès d'un large public. Celui-ci peut éventuellement les adopter peu ou prou par mimétisme, sans prendre nécessairement conscience de leur portée discursive réelle.

Dans un milieu social et discursif déterminé, le locuteur élabore ainsi sa parole à l'égard d'autrui en anticipant des réactions éventuelles selon le dialogisme interlocutif. Il fait des choix dénominatifs qui seraient a priori aptes à dire moins que prévu. Dès lors, bien que le contexte indique la réalité négative, en tant qu'occurrence linguistique ponctuelle, l'expression euphémique offre toujours la possibilité d'une interprétation fluctuante ou diffuse. Elle favorise l'atténuation, même si d'autres éléments linguistiques exercent leur influence pour la dévoiler nommant tel ou tel référent tabou. 


\section{RÉFÉRENCES BIBLIOGRAPHIQUES}

Allan, Keith \& Kate BURRIDGE (1991): Euphemism and Dysphemism. Language used as Shield and Weapon. Oxford/ New York, Oxford University Press.

AMiot, Dany \& Walter DE MuldeR (2003) : "Préposition contre préfixe ». Recherches linguistiques, 26, 203-232.

AMOSSY, Ruth (2005) : "De l'apport d'une distinction : dialogisme vs polyphonie dans l'analyse argumentative ", in Jacques Bres, Pierre P. Haillet, Sylvie Mellet, Henning Nølke \& Laurence Rosier (éd.), Dialogisme et polyphonie. Approches linguistiques. Bruxelles, De Boeck, 63-73.

ANSCOMBRE, Jean-Claude (2005) : «le ON-locuteur: une entité aux multiples visages », in Jacques Bres, Pierre P. Haillet, Sylvie Mellet, Henning Nølke et Laurence Rosier (éd.), Dialogisme et polyphonie. Approches linguistiques. Bruxelles, De Boeck, 75-94.

APOTHÉLOZ, Denis (2003) : "Le rôle de l'iconicité constructionnelle dans le fonctionnement du préfixe négatif in- ". Cahiers de linguistique analogique, 1, 35-63.

APOTHÉLOZ, Denis (2005): "Morphème opportuniste et lexicalisation d'inférences: la préfixation négative in- ». Neophilologica, 17, 84-95.

AUTHIER-REVUZ, Jacqueline (1984) : "Hétérogénéité(s) énonciative(s) ». Langages, 73, 98111.

BaKHTine, Mikhaïl (1970) : La Poétique de Dostoievski. Traduit du russe par I. Kolitcheff et présenté par J. Kristeva. Paris, Seuil. [éd. orig. : 1963].

BENETTI, Laurence et Gilles CORMINBOEUF (2004) : «Les nominalisations des prédicats d'action ". Cahiers de linguistique française, 26, 413-435.

BonHOMME, Marc (2005) : Pragmatique des figures du discours. Paris, Champion.

Bonhomme, Marc (2012) : "La réception de l'euphémisme : entre réussite et échec interactif ", in Marc Bonhomme, Mariela de la Torre \& André Horak (éd.), Études pragmatico-discursives sur l'euphémisme. Frankfurt, Peter Lang, 73-88.

BONHOMME, Marc (2020) : "Polyphonie divergente et mise en cause des euphémismes dans la presse écrite ». Çédille, revista de estudios franceses, 17 [Monografía 10 : Montserrat López Díaz \& Annabelle Seoane, éd., Les euphémismes dans les médias : entre voilements, démasquages et discours qui les traversent], 25-43. DOI: https://doi.org/10.25145/j.cedille.2020.17.03.

BERLANGA DE JESÚS, Lorenza (2000) : «Propuestas acerca del prefijo in-negativo del francés contemporáneo ». Thélème. Revista complutense de estudios franceses, 15, 193-204. Disponible sur: https://revistas.ucm.es/index.php/THEL/article/view/THEL0000110193A.

BRES, Jacques (2005) : "Savoir de quoi on parle : dialogue, dialogal, dialogique ; dialogisme, polyphonie... ", in Jacques Bres, Pierre P. Haillet, Sylvie Mellet, Henning Nølke \& Laurence Rosier (éd.), Dialogisme et polyphonie. Approches linguistiques. Bruxelles, De Boeck, 47-61. 
CONSTANTIN DE CHANAY, Hugues (2001) : "La dénomination : perspective discursive et interactive ". Cahiers de praxématique, 36, 169-188.

DAL, Georgette (2003) : «Arguments pour un préfixe "contre-" ». Recherches linguistiques, 26, 172-201.

DÉTRIE, Catherine; Paul SIBLOT \& Bertrand VÉRINE (2001): Termes et concepts pour l'analyse du discours. Une approche praxématique. Paris, Champion.

GreIMAS, Algirdas Julien (1966) : Sémantique structurale. Paris, Larousse.

HUYGHE, Richard (2014) : «La sémantique des noms d'action : quelques repères ». Cahiers de lexicologie, 105/2, 181-201.

JAUBERT, Anna (2008) : " Dire et plus ou moins dire. Analyse pragmatique de l'euphémisme et de la litote ». Langue française, 160, 105-116.

LECOlLE, Michèle; Marie VeniaRd \& Olivia GuÉRIN (2018) : "Pour une sémantique discursive : propositions et illustrations ». Langages, 210, 35-54.

LÓPEZ DÍAZ, Montserrat (2016) : «Euphémismes néosémiques au sujet de l’emploi en temps de crise ». La Linguistique, 52/2, 239-256.

LÓPEZ DÍAZ, Montserrat (2018) : "La cooccurrence du tabou et de l'euphémisme ou les conditions de la synonymie ». Travaux de linguistique, 76, 27-42.

LÜDI, Georges (1995) : "Représentations lexicales floues et construction interactive du sens ». Cahiers de l'ILSL, 7, 95-109.

MOIRAND, Sophie (2007) : Les Discours de la presse quotidienne. Paris, PUF.

MOIRAND, Sophie (2010) : "Retour sur une approche dialogique du discours ", in Marion Colas-Blaise, Mohamed Kara, Laurent Perrin \& André Petitjean (éd.), La Question polyphonique ou dialogique en sciences du langage. Metz, CELTED-Recherches linguistiques, 375-398.

MORIER, Henri (1998) : Dictionnaire de poétique et de rhétorique. Paris, PUF. [1éd. 1961].

NOWAKOWSKA, Aleksandra (2005) : "Dialogisme, polyphonie: des textes russes de M. Bakhtine à la linguistique contemporaine ", in Jacques Bres, Pierre P. Haillet, Sylvie Mellet, Henning Nølke \& Laurence Rosier (éd.), Dialogisme et polyphonie. Approches linguistiques. Bruxelles, De Boeck, 19-32.

RABATEL, Alain (2006) : «La dialogisation au cœur du couple polyphonie / dialogisme chez Bakhtine ». Revue Romane, 41/1, 55-80.

RASTIER, François (1985) : "L'isotopie sémantique, du mot au texte ». L'Information grammaticale, 27, 33-36.

SEOANE, Annabelle (2016) : «Deux néologismes par glissement sémantique : quand l'euphémisme cristallise ». La Linguistique, 52/2, 271-290.

SiblOT, Paul (2001) : "De la dénomination à la nomination. Les dynamiques de la signifiance nominale et le propre du nom ». Cahiers de praxématique, 36, 189-214.

STAAFF, Erik (1928), "Étude sur les mots composés avec le préfixe négatif in- en français ». Studia Neophilologica, 1/1, 45-73. 
TODOROv, Tzvetan (1981): Mikhaïl Bakhtine. Le principe dialogique suivi de Écrits du Cercle. Paris, Seuil.

Trésor de la langue française. Dictionnaire de la langue du XIXe et du XXe siècle. París, CNRS, 1971-1994. Version en ligne: ATILF-CNRS \& Université de Lorraine. Disponible sur : http://atilf.atilf.fr/tlf.htm.

VAN GOETHEM, Kristel (2006) : "L'emploi "préfixal" des prépositions contre et tegen. Une analyse contrastive ". Travaux de linguistique, 52, 115-145.

VION, Robert (2010) : "Polyphonie énonciative et dialogisme", in Colloque international Dialogisme: langue, discours. Université de Montpellier. Disponible sur: https://www.praxiling.fr/spip.php?action=acceder_document\&arg=538\&cle=e618d4be7cb7 6beeee29952c23f4c299231154cc\&file=pdf_Vion1.pdf. 